INHALT

VOR WORT der Herausgeber.

\title{
EINFÜHRUNG
}

\section{REIMER HANSEN}

Die wissenschaftsgeschichtlichen Zusammenhänge der Entstehung und der Anfänge der modernen Geschichtswissenschaft ....... 3

WOLFGANG RIBBE

Berlin als Standort historischer Forschung

\section{BEITRÄGE}

HEINZ GRÜNERT

Ur- und Frühgeschichtsforschung in Berlin .

ALEXANDER DEMANDT

Alte Geschichte in Berlin 1810-1960

KASPAR ELM

Mittelalterforschung in Berlin. Dauer und Wandel

DIETER HERTZ-EICHENRODE

Die „Neuere Geschichte“ an der Berliner Universität. Historiker und Geschichtsschreibung im 19./20. Jahrhundert... 


\section{GERD HEINRICH}

Brandenburgische Landesgeschichte und preußische Staatsgeschichte. Universitäten, Hochschulen, Archive. Historische Gesellschaften und Vereine

ECKART HENNING

Die Historischen Hilfswissenschaften in Berlin

KURT-VICTOR SELGE

Die Berliner Kirchenhistoriker

FRIEDRICH EBEL

Rechts- und Verfassungsgeschichte in Berlin im 19. und 20. Jahrhundert

WOLFRAM FISCHER

Sozial- und Wirtschaftsgeschichte in Berlin

MARIANNE AWERBUCH

Die Hochschule für die Wissenschaft des Judentums

KLAUS MEYER

Osteuropäische Geschichte

KLAUS ZERNACK

"Deutschland und der Osten" als Problem der historischen For-

schung in Berlin

WILLI PAUL ADAMS

Die Geschichte Nordamerikas in Berlin

REINHARD LIEHR

Geschichte Lateinamerikas in Berlin

HAGEN SCHULZE

Entwürfe Historischer Welten. Von Humboldt bis Meinecke .. 657 
CAROLINE FLICK und GERTRAUD SCHRAGE

Auswahlbibliographie ........................... 677

ROSEMARIE BAUDISCH

Register (Personen und Institutionen) ............... 847 
